\title{
Ganho de Conhecimento no Internato Médico em Psiquiatria Não Reduz Estigmatização dos Transtornos Mentais
}

\author{
Increased Knowledge among Psychiatric \\ Medical Interns does not Reduce the \\ Stigmatization of Mental Disorders
}

\author{
Gabriel de Oliveira Araújo \\ Mariana Mendonça Ferreira Ramos ${ }^{I}$ \\ Antonio Pedro de Melo Moreira Suarte \\ Lays Genro Coutinho \\ Bárbara Vidigal Braga \\ Thiago Blanco-Vieira ${ }^{\text {I॰ }}$
}

PALAVRAS-CHAVE

- Estigma.

- Saúde Mental.

- Educação Médica.

- Internato.

- Currículo Médico. 


\section{KEY-WORDS}

- Social Stigma.

- Mental Health

- Medical Education.

- Clinical Internship.

- Curriculum.

\begin{abstract}
Introduction: Considering the high prevalence of mental illnesses, it is essential for any physician to offer proper treatment and attention to people with these disorders. Nevertheless, the usual stigma and lack of knowledge regarding the management of mental disorders on the part of physicians can represent a significant barrier to treatment and inefficiency faced by patients in the health care system. Objective: This study aimed to measure the gain of psychiatric knowledge and the reduction in the stigmatization of people with schizophrenia by medical students after exposure to the compulsory internship program at a public medical school in the Federal District. Methods: Quasi-experimental study to evaluate the impact of the training program during medical internship in the final year of undergraduate medical training. The sample was 35 final-year medical students. Questionnaires were applied to measure the degree and type of stigma in relation to schizophrenia and self-perception regarding the drug management and treatment of psychiatric diseases. A three-point Likert scale was used to measure the results. The questionnaires were applied immediately before and after exposure to the four-week training program. The medical values of self-perception and stigma were compared between two moments using the Student's t test. Results: There was no statistically significant difference found between stigmatization before and after the intervention (total stereotype, $p=0.230$; perceived prejudice, $p=0.172$; social distance, $p=0.209$; civil rights, $p=0.837$ ). Regarding selfperception of knowledge, the number of items reaching total confidence and the mean values after the training were significantly improved ( $p=0.007$ and $p<0.0001$, respectively). There was no correlation with sociodemographic characteristics. Conclusion: Despite the knowledge gain, medical students failed to show any improvement in the degree of stigma held after immersion in a 4-week practical psychiatric training program. The short duration of the intervention might have meant that the students' contact with patients was insufficient to reduce stigma. More studies are needed with larger groups and experimental designs.
\end{abstract}

Recebido em: 18/6/19

Aceito em: 3/8/19

\section{INTRODUÇÃO}

Os transtornos mentais estão entre as condições de saúde de maior morbidade, alcançando taxas de prevalência estimadas ao longo da vida de $25 \%$ da população geral mundial ${ }^{1}$. Dada a relevância dessa epidemiologia, o cuidado em saúde eficiente para essas condições deve ser competência essencial de qualquer médico, sobretudo daqueles dedicados à atenção primária $^{1,2}$.

O ensino da psiquiatria nas escolas médicas, no entanto, usualmente é descrito como breve e desproporcional à magnitude da frequência dos transtornos mentais ${ }^{3}$. Essa imperfeição nos currículos dos cursos médicos é uma das importantes causas de um grave hiato terapêutico na saúde mental existente na atenção básica, em virtude da formação de médicos mal preparados para diagnosticar e tratar a maioria dos problemas de saúde mental ${ }^{4}$.

Em conjunto, a falta de conhecimento no manejo das doenças mentais e o estigma relacionado à doença mental por parte dos médicos podem representar uma barreira intransponível enfrentada pelos pacientes em todos os níveis de atenção ${ }^{5}$.

A graduação médica representa um espaço valioso para a melhora desse cenário crítico ${ }^{3}$. Segundo Yadav et al. ${ }^{6}$, o treinamento em serviço em psiquiatria tem alto potencial de produzir resultados satisfatórios para o desenvolvimento de atitudes positivas frente à doença mental entre estudantes de Medicina e, por conseguinte, entre os profissionais formados.

Após a exigência da inclusão da saúde mental como área básica nos parâmetros curriculares nacionais dos cursos de Medicina ${ }^{7}$, as escolas médicas estão desde então obrigadas a incluir o treinamento em saúde mental no internato. No entanto, a escassez de publicações sobre a eficácia destas intervenções reforça a importante falha percebida na cultura institucional e educacional em avaliar sistematicamente a eficácia dos programas educacionais ${ }^{8}$.

Considerando que não há lugar na gestão de recursos públicos para a adoção de qualquer iniciativa que não tenha 
sua eficácia devidamente comprovada ${ }^{9}$, a implementação de estratégias de avaliação de impacto de programas de treinamento é essencial.

Seguindo esta perspectiva, este estudo avaliou o impacto do treinamento obrigatório em psiquiatria na modalidade internato médico nos domínios de conhecimento e estigmatização de pessoas portadoras de transtorno mental entre estudantes do último ano do curso médico. A hipótese dos autores é de que a imersão com enfoque clínico no manejo de pacientes portadores de transtorno mental em serviços especializados seja suficiente para reduzir preconceitos e distanciamento social, além de aprimorar as competências clinicas na abordagem e tratamento das pessoas em sofrimento psíquico.

O estudo tem por objetivo fomentar dados que permitam a elaboração de programas de treinamento no internato do curso médico que de fato tenham resultado favorável na formação profissional dos futuros médicos.

\section{MÉTODO}

\section{Desenho do estudo}

Foi realizada uma avaliação com desenho quasi-experimental. A avaliação da estigmatização e da competência foi feita antes e após a execução do programa de treinamento por imersão em serviço de psiquiatria por estudantes do último ano do curso de Medicina de uma instituição de ensino superior pública no Distrito Federal.

A pesquisa foi aprovada pelo Comitê de Ética e Pesquisa da Fundação de Ensino e Pesquisa em Ciências da Saúde (Fepecs) (registro ${ }^{\circ}$ CAAE 80614617.5.0000.5553).

\section{Descrição do programa educacional}

O programa avaliado compreende um dos estágios curriculares obrigatórios do currículo do curso médico na modalidade internato médico. O programa consistiu numa imersão em serviço, com duração de quatro semanas, perfazendo 40 horas por semana de atividades em serviços especializados em saúde mental, com grupos de oito a dez estudantes do curso de Medicina (Quadro 1).

A metodologia aplicada no treinamento utilizou os seguintes recursos pedagógicos/cenários de aprendizagem:

(a) Discussões de casos clínicos e seminários sobre assuntos referentes a: semiologia psiquiátrica e especificidades da entrevista médica, abordagem do paciente e fenomenologia aplicada à saúde mental; transtornos do humor; transtornos do espectro da esquizofrenia; transtornos ansiosos; farmacologia dos antidepressivos e estabilizadores do humor; farmacologia dos ansiolíticos e hipnóticos; emergências psiquiátricas; psiquiatria do desenvolvimento com ênfase nas doenças mais prevalentes na infância e adolescência (autismo, transtorno do déficit de atenção e hiperatividade e transtorno de oposição e desafio/transtorno de conduta) e padrões de manejo parental; manejo medicamentoso de populações especiais: idosos, gestantes, crianças e portadores de doenças crônicas (oncológicas e pessoas vivendo com HIV, por exemplo); políticas públicas em saúde mental. $\mathrm{O}$ recurso pedagógico adotou integralmente a aplicação de técnicas de problematização com base em casos clínicos vivenciados pelos estudantes. Os temas abordados foram distribuídos em três encontros semanais sob orientação de um professor especialista em psiquiatria. A duração de cada encontro foi de quatro horas;

(b) Ambulatórios de psiquiatria no hospital geral, em centros de atenção psicossocial para tratamento de pessoas com transtornos relacionados ao uso de substâncias (CAPSad) e para pessoas portadoras de transtornos mentais gerais (CAPS);

(c) Emergência psiquiátrica em hospital geral e em hospital psiquiátrico;

(d) Interconsulta psiquiátrica, definida pelo atendimento de pacientes hospitalizados em leitos de outras especialidades médicas que requerem suporte pela psiquiatria.

\section{Participantes}

Foram convidados e incluídos 34 alunos do curso de Medicina do sexto ano no primeiro semestre de 2018. Não houve recusa em participar do estudo. Todos os participantes assinaram o Termo de Consentimento Livre e Esclarecido, obtido por escrito.

Os critérios de exclusão foram: estar envolvido diretamente com a pesquisa; ter participação prévia em liga acadêmica de psiquiatria; ter cursado no mínimo $60 \%$ de graduação prévia em curso superior na área de saúde mental; ausentar-se em qualquer etapa de aplicação dos instrumentos.

\section{Instrumentos}

Foram utilizados dois instrumentos:

1. Questionário para avaliação de estigmatização de pacientes portadores de esquizofrenia, desenvolvido por Loch et al. ${ }^{10}$. O instrumento contém duas partes, descritas a seguir:

(a) Padrão e natureza do contato com a doença mental. A variável de interesse neste quesito correspondeu à identificação da presença de um membro da família e/ou conhecido com transtorno psiquiátrico e, quando afirmativo, a frequência de contato com esse indivíduo. As opções de frequência eram: diariamente, semanalmente, mensalmente, ocasionalmente e nunca.

(b) Dimensões de estigma. O questionário compõe-se de quatro dimensões do estigma: estereótipos (12 características para serem comparadas com as de alguém da população 
QuAdro 1

\section{Descrição das atividades curriculares do internato em psiquiatria da ESCS}

$\begin{array}{lc}\text { Atividade } & \text { Carga horária } \\ \text { Programa teórico } & 48 \mathrm{~h} \\ \end{array}$

Ambulatórios de psiquiatria no

hospital geral

Centros de Atenção Psicossocial para tratamento de pessoas com transtornos relacionados ao uso de substâncias (CAPSad)

Centros de Atenção Psicossocial para tratamento de pessoas portadoras de transtornos mentais gerais (CAPS)

Emergência psiquiátrica 9

Interconsulta psiquiátrica

\section{Descrição}

Discussão de casos clínicos e seminários sobre assuntos referentes a: semiologia psiquiátrica e especificidades da entrevista médica, abordagem do paciente e fenomenologia aplicada à saúde mental; transtornos do humor; transtornos do espectro da esquizofrenia; transtornos ansiosos; farmacologia dos antidepressivos e estabilizadores do humor; farmacologia dos ansiolíticos e hipnóticos; emergências psiquiátricas; psiquiatria do desenvolvimento com ênfase nas doenças mais prevalentes na infância e adolescência (autismo, transtorno do déficit de atenção e hiperatividade e transtorno de oposição e desafio/transtorno de conduta) e padrões de manejo parental; manejo medicamentoso de populações especiais: idosos, gestantes, crianças e portadores de doenças crônicas (oncológicas e pessoas vivendo com HIV, por exemplo); políticas públicas em saúde mental.

Ambulatórios executados por médico psiquiatra em três diferentes hospitais da rede SUS do Distrito Federal; os ambulatórios eram de psiquiatria geral, caracterizando modelo de ambulatório de especialidade.

Atividade assistencial executada por médico psiquiatra especializado em transtorno relacionado ao uso de substâncias, entrevista com pacientes em leito de permanência, grupo de medicação e grupo de manejo de tabagismo. Nos grupos de terapia, são realizadas dinâmicas e espaços para troca de experiências entres os pacientes. Os estudantes auxiliam os profissionais de saúde mental a coordenar os eventos.

$8 \mathrm{~h} \quad$ Atividade assistencial executada por médico psiquiatra para manejo de pacientes graves que requerem assistência em regime intensivo ou semi-intensivo.

$9 \mathrm{~h} \quad$ Atendimento de pacientes portadores de transtorno mental ou em situação de sofrimento psíquico, em crise.

$8 \mathrm{~h} \quad$ Atendimento de pacientes hospitalizados em leitos de outras especialidades médicas que requerem suporte pela psiquiatria.

geral); preconceito percebido (opinião do entrevistado sobre nove afirmações relacionadas a atitudes da população em geral para com o indivíduo índice), distância social (mede a relutância do entrevistado em participar de certas atividades com o indivíduo índice) e restrições de direitos civis. A aferição do estigma foi gerada pela opinião que o participante deveria manifestar em relação a um indivíduo descrito numa vinheta de abertura da entrevista. Nesta, o instrumento descrevia um indivíduo adulto, jovem, do sexo masculino, com quadro psicopatológico de esquizofrenia. A opinião dos participantes foi aferida durante todo o questionário por meio de escala do tipo Likert de três pontos. A fim de evitar viés de aferição, os itens admitiam pontuação invertida. Nesses casos, as pontuações foram corrigidas para a apreciação dos dados. Em todos os itens, os participantes tinham a opção de escolher as seguintes opções, que foram consideradas pontuação nula: "prefiro não responder" ou "não sei responder". Para avaliar estereótipos, ambos os estereótipos - positivos (referentes aos itens que continham características positivas, como ser mais criativo ou talentoso) e negativos (referentes aos itens com características negativas, como ser pouco inteligente, perigoso ou não confiável) - foram aferidos pelo instrumento. A soma total dos estereótipos positivos e dos negativos poderia variar de 12 (mí- nimo de estigmatização) a 36 (máximo de estigmatização). $\mathrm{O}$ preconceito percebido (percepção de atitudes estigmatizantes pela sociedade em geral, pela maioria das pessoas) foi avaliado por meio de nove itens, baseados nas escalas de aceitação social e de estigmatização social. Ambas as escalas medem as atitudes sociais em relação às pessoas com esquizofrenia. A distância social foi avaliada com uma adaptação da escala de distância social ${ }^{9}$, por meio de um grupo de sete perguntas que mediam a relutância do entrevistado em participar de certas atividades sociais com o suposto indivíduo portador de transtorno mental da vinheta descrita. O item "você alugaria um quarto para uma pessoa com esquizofrenia?" foi substituído por "você convidaria uma pessoa com esquizofrenia para uma festa, uma reunião ou um jantar?", já que no Brasil é bastante incomum alugar um quarto para outra pessoa. A restrição de direitos civis foi avaliada por meio de três itens (autonomia para escolha de tratamento, capacidade de votar e dirigir).

2. Questionário elaborado para aferição da autopercepção dos conhecimentos, elaborado pelos autores. $\mathrm{O}$ instrumento foi composto por 12 itens com aferição de resposta do tipo Likert de três pontos que avaliam autopercepção sobre manejo de medicamentos e sobre tratamento de doenças psiquiátricas de impacto epidemiológico. 


\section{Análise de dados}

Os valores médios de autopercepção e estigma foram comparados entre os dois momentos, início do estágio e final do estágio, empregando-se o teste $\mathrm{t}$ de Student emparelhado. As correlações das medidas de autopercepção com estigma foram analisadas empregando-se o coeficiente de correlação linear de Pearson. As associações das variáveis qualitativas demográficas com o ganho em autopercepção e com o ganho na escala de estigma, medido pela diferença dos escores no momento pós- em relação ao pré-, foram realizadas pelo teste qui-quadrado. As análises foram conduzidas pelo aplicativo SAS 9.4. Considerou-se significativo um valor de $p<0,05$.

\section{RESULTADOS}

Os participantes foram prioritariamente homens $(n=22,65 \%)$, com distribuição etária similar entre idade superior $(n=16$, $47 \%$ ) e inferior ou igual a 25 anos $(n=18,53 \%)$. A maior parte dos participantes identificou-se como vinculada à religião católica ( $\mathrm{n}=14,41 \%$ ), mas outras religiões também foram elencadas: evangélica ( $n=9,26 \%$ ), espírita ( $n=5,15 \%)$ e outras religiões não especificadas ou sem vinculação à religião (n = $6,18 \%)$.

A intervenção educacional demonstrou eficácia estatisticamente significativa na avaliação da percepção de competência para manejo e tratamento dos transtornos mentais. Os participantes obtiveram aumento tanto no número de respostas para as quais se sentiam plenamente confiantes em sua capacidade quanto na melhora da pontuação geral, que indica maior grau de competência percebida após a intervenção $(\mathrm{p}=$ 0,0074 e $\mathrm{p}<0,0001$, respectivamente) (Tabela 1 ).

\begin{tabular}{|c|c|c|c|}
\hline \multicolumn{4}{|c|}{$\begin{array}{c}\text { TABELA } 1 \\
\text { Valores médios da autopercepção nos } \\
\text { momentos pré- e pós-teste }\end{array}$} \\
\hline Autopercepção & Pré- & Pós- & p valor ${ }^{\#}$ \\
\hline $\begin{array}{l}\text { Itens de confiança máxima } \\
(\text { máx. }=11)\end{array}$ & $0,85 \pm 1,89$ & $2,53 \pm 2,95$ & 0,007 \\
\hline $\begin{array}{l}\text { Pontuação de confiança } \\
(\text { máx. = 33) }\end{array}$ & $16,44 \pm 4,93$ & $23,15 \pm 3,58$ & $<0,0001$ \\
\hline
\end{tabular}

Não houve mudança estatisticamente significativa entre as somas das pontuações de estigma total ( $p=0,1313)$. As dimensões de estereótipo $(\mathrm{p}=0,230)$, preconceito percebido $(\mathrm{p}=$ $0,172)$, distância social $(\mathrm{p}=0,209)$ e restrição de direitos civis $(\mathrm{p}=0,837)$ não apresentaram diferença estatisticamente significativa entre os dois momentos (Tabela 2).

\section{TABELA 2}

Valores médios de estigma nos momentos pré- e pós-teste

\begin{tabular}{lccc} 
& \multicolumn{1}{c}{ Pré- } & \multicolumn{1}{c}{ Pós- } & $p$ \\
Estereótipos & $16,56 \pm 5,85$ & $18,18 \pm 4,31$ & 0,230 \\
Preconceito percebido & $10,82 \pm 3,89$ & $11,88 \pm 3,46$ & 0,172 \\
Distância social & $13,85 \pm 3,99$ & $14,94 \pm 3,79$ & 0,209 \\
Restrições de direitos civis & $6,35 \pm 1,01$ & $6,29 \pm 1,47$ & 0,837 \\
Total & $47,59 \pm 10,71$ & $51,29 \pm 7,65$ & 0,131
\end{tabular}

*Valores expressos em média \pm desvio padrão; ${ }^{* *} p$-valor calculado pelo teste $t$ de Student emparelhado.

Não houve correlação estatisticamente significativa entre a autopercepção de conhecimento específico e as dimensões de estigma avaliadas. Portanto, maior conhecimento neste estudo não esteve correlacionado com redução do estigma dos estudantes em relação aos portadores de transtorno psicótico (Tabela 3).

Nenhuma das variáveis sociodemográficas pesquisadas esteve estatisticamente correlacionada com mudança no grau de confiança percebido pelos participantes.

\section{DISCUSSÃO}

Este é o primeiro estudo que avaliou o impacto do programa de imersão em psiquiatria no internato do curso médico no tocante ao grau de estigmatização e sua relação com a percepção de competência pelos estudantes. Os resultados apontam que o programa descrito teve êxito na melhora de percepção de competência clínica com o treinamento, porém tal ganho se mostrou insuficiente para mudar o grau de estigmatização em relação ao paciente com transtorno mental psicótico.

O ganho em autoconfiança representa um aspecto relevante no tocante à avaliação de eficácia do programa educacional. Essa variável configura-se como um indicador indireto de melhora no desempenho profissional, pois, sentindo-se

\begin{tabular}{|c|c|c|c|c|c|}
\hline \multicolumn{6}{|c|}{$\begin{array}{r}\text { TABELA } 3 \\
\text { Correlação entre total do estigma e a soma dos itens da a }\end{array}$} \\
\hline & & \multicolumn{4}{|c|}{ Estigma } \\
\hline & & pré-teste & $p$ & pós-teste & $p$ \\
\hline \multirow{2}{*}{ Autopercepção } & pré-teste & $0,311(-0,030-0,588)$ & 0,069 & - & \\
\hline & pós-teste & - & & $0.311(-0,030-0,588)$ & 0,069 \\
\hline
\end{tabular}


mais autoconfiante para executar determinada tarefa, o indivíduo tende a manifestar maior disponibilidade para o cuidado e a ter melhores resultados globais ${ }^{11}$.

No entanto, houve uma insuficiência do programa em reduzir o nível de estigmatização em relação aos pacientes portadores de transtorno psicótico. Os autores acreditam que o tempo de exposição dos estudantes ao contato com os pacientes portadores de transtorno mental nesse programa possa ter sido insuficiente, o que também foi apontado como potencial causa de insucesso na melhora do estigma em estudos prévios ${ }^{12-14}$.

Esperava-se que a adoção de elementos já apontados como importantes para o sucesso de programas educacionais em saúde mental, como metodologias ativas de ensino-aprendizagem, abordagem de temas e conteúdos relacionados à prática diária do treinamento dos estudantes e envolvimento de docentes especialistas tanto na elaboração quanto na execução do programa ${ }^{8,15}$, fossem suficientes para produzir ganhos nas duas dimensões.

Estudos prévios indicam ainda uma relação inversa entre ter contato com pessoas portadoras de doença mental e grau de estigmatização pelas pessoas ${ }^{16}$. Portanto, um tempo maior de contato com os doentes parece ser uma estratégia fundamental para sanar tal insuficiência nos processos de formação da graduação médica.

Curiosamente, mais do que apenas não reduzir o estigma, o contato breve com pacientes esquizofrênicos pode piorar o estigma por médicos em estágios tão curtos como quatro semanas em psiquiatria ${ }^{14}$. Acredita-se que o tempo insuficiente impeça que o estudante observe melhora ou recuperação dos pacientes com doenças psiquiátricas, o que pode ter impacto na manutenção do estigma ${ }^{12-14}$.

Apesar da superioridade das metodologias ativas de ensino-aprendizagem no ganho de conhecimento, habilidades e atitudes de estudantes comparativamente ao modelo tradicional de ensino, tal modalidade pedagógica não tem alcance na diminuição do estigma por estudantes ${ }^{17}$. Logo, a despeito do uso de bons recursos pedagógicos, é preciso adotar outras estratégias para aperfeiçoar as práticas educacionais correntes.

Algumas limitações deste estudo precisam ser consideradas:

(a) O método de autorrelato por meio do qual as informações foram coletadas, uma amostra por conveniência, o tamanho reduzido da amostra e a origem dos estudantes (de apenas uma escola médica) limitam a capacidade de generalização do estudo; no entanto, a entrevista face a face poderia ter produzido constrangimento involuntário e, dessa forma, ter influenciado as respostas, estimulando respostas socialmente desejáveis e distorcendo a avaliação do estigma; (b) Ausência de grupo controle de estudantes da mesma seriação, sobretudo por questões logísticas. Além disso, considerando que os rodízios do internato são independentes, não seria possível controlar exatamente se o estudante de outro rodízio poderia ter tido acesso a recursos de aprendizagem em saúde mental mesmo não sendo aquela a competência básica de objetivo do rodízio. No entanto, considerando que, seja qual for a especialidade médica, haverá contato com pacientes em sofrimento psíquico, podemos supor que outras variáveis poderiam influenciar e deflagrar viés no grupo controle;

(c) Fatores relevantes do ponto de vista sociodemográfico não foram pesquisados, como renda, orientação sexual e frequência de contato com pessoas portadoras de transtorno mental no convívio usual do participante;

(d) a ausência de um instrumento padronizado para aferição do estigma e conhecimento em saúde mental, justificada pela ausência de um questionário validado para este propósito no Brasil, compromete a validade externa do estudo. Como estratégia para minimizar este obstáculo, optou-se pelo instrumento desenvolvido na Universidade de São Paulo (USP) por Loch et al. ${ }^{9}$ devido à sua maior abrangência de dimensões do estigma e aceitabilidade do instrumento. Cabe apontar que o estudo desses autores foi realizado com uma população de médicos psiquiatras, e este estudo avaliou uma população de estudantes do curso de Medicina. Essa diferença etária entre as amostras pode limitar o poder de comparação entre os grupos;

(e) A quantidade de conteúdo teórico com diversos temas dentro do programa do internato pode ser um fator de não aprofundamento no tema esquizofrenia, que é o principal tema abordado pelo questionário que avalia estigma.

Independentemente das limitações, este estudo representa uma iniciativa inovadora no campo da educação médica no Brasil tanto por aplicar método de avaliação de eficácia de intervenção educacional quanto para a elaboração de intervenções pedagógicas em saúde mental na graduação médica que alcancem desfechos mais robustos do que apenas ganho de conhecimento. E isso é, indubitavelmente, um desfecho valioso para a formação profissional eficaz e para o cuidado eficiente em saúde.

\section{CONCLUSÃO}

Alinhado com estudos prévios, o treinamento por imersão em serviço, característico dos programas de internato no curso médico, não demonstrou evidência de capacidade de redução do estigma. Esses achados ainda preliminares indicam que é necessária uma revisão do currículo médico de forma a assegurar que haja redução do grau de estigmatização dos pacientes portadores de transtorno mental de forma a garantir a formação de médicos não apenas capazes tecnicamente, 
mas qualificados nas competências ligadas às humanidades. Estudos futuros com adoção de estratégias inovadoras de ensino-aprendizagem e maior tempo dedicado ao internato em saúde mental poderão oferecer respostas complementares aos achados do presente estudo.

\section{REFERÊNCIAS}

1. World Health Organization. The World Health report 2001: Mental Health: new understanding, new hope. Geneva: World Health Organization; 2001. (Relatório)

2. Kohrt B, Asher L, Bhardwaj A, Fazel M, Jordans M, et al. The role of communities in mental health care in low-and middle-income countries: a meta-review of components and competencies. International journal of environmental research and public health 2018; 15(6) 1279.

3. Schlozman S, Beresin EV, Balon R, Coverdale JH, Brenner AM, Louie AK, et al. Stigma and Mental Health: A Proposal for Next Steps. Academic Psychiatry 2016; 40(5) 735-39.

4. Saxena S, Thornicroft G, Knapp M, Whiteford H. Resources for mental health: scarcity, inequity, and inefficiency. The lancet 2007; 370(9590) 878-889.

5. Arizaga MA, Eizaguirre CS, Feliu MR, Etxeberria AS. 2004. Actitudes Del PersonalSanitario de Atención Primaria Hacia El Enfermo Mental, La Psiquiatría y El Equipo de Salud Mental. Aten Primaria 2004; 33(9) 491-495.

6. Yadav T, Arya K, Kataria D, Balhara YPS. Impact of Psychiatric Education and Training on Attitude of Medical Students towards Mentally Ill: A Comparative Analysis. Industrial PsychiatryJournal 2012; 21(1) 22-31.

7. Conselho Nacional de Educação. Diretrizes curriculares nacionais para cursos de medicina. Brasília: Ministério da Educação 2014.

8. Blanco-Vieira T, da Cunha Ramos FA, Lauridsen-Ribeiro E, Ribeiro MVV, Meireles, EA, Nóbrega BA, et al. A Guide for Planning and Implementing Successful Mental Health Educational Programs. Journal of Continuing Education in the Health Professions 2018; 38(2) 126-136.

9. Lock K. Health impact assessment. BMJ 2000;320 1395-1398.

10. Loch AA, Guarniero FB, Lawson FL, Hengartner MP, Rössler W, Gattaz WF, et al. Stigma toward Schizophrenia: Do All Psychiatrists Behave the Same? Latent Profile Analysis of a National Sample of Psychiatrists in Brazil. BMC psychiatry 2013; 13(92) 1-10.

11. Baumeister RF, Campbell JD, Krueger JI, Vohs KD. Does High Self-Esteem Cause Better Performance, Interpersonal Success, Happiness, or Healthier Lifestyles?. Psychol Sci Public Interest 2003; 4(1) 1-44.
12. Mino Y, Yasuda N, Kanazawa S, Inoue S. Effects of Medical Education on Attitudes towards Mental Illness among Medical Students: A Five-Year Follow-up Study. Acta Medica Okayama 2000; 54(3) 127-132.

13. Wang PW, Ko CH, Chen CS, Yang YC, Lin HC, Cheng CC, et al. Changes of Explicit and Implicit Stigma in Medical Students during Psychiatric Clerkship. Academic Psychiatry 2016; 40(2) 224-228.

14. Omori A, Tateno A, Ideno T, Takahashi H, Kawashima Y, Takemura K, et al. "Influence of Contact with Schizophrenia on Implicit Attitudes towards Schizophrenia Patients Held by Clinical Residents. BMC Psychiatry 2012; 12(205) 1-8.

15. Blanco-Vieira T, Ribeiro WS, Lauridsen-Ribeiro E, de Sá DGF, Palma SMM, Ribeiro MVV, et al. An evaluation of a collaborative course for child and adolescent mental health professionals. Journal of interprofessional care 2017; 31(5) 664-666.

16. Morgan AJ, Reavley NJ, Ross A, Too LS, Jorm AF. Interventions to reduce stigma towards people with severe mental illness: Systematic review and meta-analysis. Journal of psychiatric research 2018; 103 120-133.

17. McParland M, Noble LM, Livingston G,McManus C. The effect of a psychiatric attachment on students' attitudes to and intention to pursue psychiatry as a career. MedEduc2003;37:447-54.

\section{CONTRIBUIÇÃO DOS AUTORES}

Todos os coautores participaram de todas as etapas do projeto, desde seu delineamento, revisão da literatura e coleta de dados até análise dos resultados. A redação do manuscrito foi realizada com a participação de todos, com destaque para os dois primeiros autores. Ao último coube o acompanhamento do processo de redação do texto e a revisão final de linguagem e conteúdo. Todos os autores aprovaram a versão final a ser submetida.

\section{CONFLITO DE INTERESSES}

Os autores declaram não haver conflito de interesses.

\section{ENDEREÇO PARA CORRESPONDÊNCIA}

Thiago Blanco-Vieira

Escola Superior de Ciências da Saúde - Curso de Medicina

Setor Médico Hospitalar Norte, Conjunto A, Bloco 01, Edifício Fepecs

Asa Norte - Brasília

CEP 70710-907 - DF

E-mail: thiagoblanco.psiquiatria@gmail.com 